\title{
Review of some recent advances on filtration in pharmaceutical industry
}

\author{
S. O. Majekodunmi*1 and E. O. Olorunsola \\ Department of Pharmaceutics and Pharmaceutical Technology, Faculty of Pharmacy, University of Uyo, Uyo, \\ Nigeria
}

\begin{abstract}
Summary: Filtration is the removal of solids suspended in a liquid or gas by passage through a pervious medium on which the solids are retained. This review focuses on some advantages in different types of filters, sintered metal filters, nanofiltration, and their applications. Some advances in bag and belt filtration and advantages of self cleaning filters over the traditional manual and mechanical cleaning as well as fermentation, water purification and sterile filtration were also discussed. Thus advances in filtration technology include the development of continuous processes to replace old technology. Different self cleaning filters reduce product loss, improve flow consistency and require minimal operators' impute. Thus, by using latest filters and filter media in pharmaceutical industry, time is reduced and better quality products are obtained.
\end{abstract}

Keywords: ultra filtration, filter media, bag filtration, belt filtration

\section{Intoduction}

Filtration is a process whereby solid particles present in a suspension are separated from the liquid or gas employing a porous medium, which retains the solids but allows the fluid to pass through [1]. When the proportion of solids in a liquid is less, the term clarification is used. It is a common operation which is widely employed in the production of sterile products, bulk drugs, and in liquid oral formulation. The suspension to be filtered is known as slurry. The porous medium used to retain the solids is known as filter medium. The accumulated solids on the filter are referred as filter cake and the clear liquid passing through the filter is filtrate. The pores of the filter medium are smaller than the size of particles to be separated. Therefore, solids are trapped on the surface of the filter medium. After a particular point of time, the resistance offered by the filter cake is high that stops the filtration [2].

Filtration is also used to describe some biological processes, especially in water treatment and sewage treatment in which undesirable constituents are removed by absorption into a biological film grown on or in the filter medium as in slow sand filtration.

\subsection{Application of filtration in Pharmaceutical industry}

Filtration is used to separate particles and fluid in a suspension, where the fluid can be a liquid, a gas or a supercritical fluid. Depending on the application, either one or both of the components may be isolated.

Filtration, as a physical operation is very important in chemistry for the separation of materials of different chemical composition. A solvent is chosen which dissolves one component, while not dissolving the other. By dissolving the mixture in the chosen solvent, one component will go into the solution and pass through the filter, while the other will be retained. This is one of the most important techniques used by chemists to purify compounds.

Filtration is also important and widely used as one of the unit operations of pharmaceutical technology. It may be simultaneously combined with other unit operations to process the feed stream, as in the bio-filter, which is a combined filter and biological digestion device.

Filtration differs from sieving, where separation occurs at a single perforated layer (a sieve). In sieving, particles that are too big to pass through the holes of the sieve are retained as in particle size distribution. In filtration, a multilayer lattice retains those particles that are unable to follow the tortuous channels of the filter. Oversize particles may form a cake layer on top of the filter and may also block the filter lattice, preventing the fluid phase from crossing the filter. Filtration differs from adsorption, where it is not the physical size of particles that causes separation but the effects of surface charge. Some adsorption devices containing activated charcoal and ion exchange resin are commercially called filters, although filtration is not their principal function.

Filtration also differs from removal of magnetic contaminants from fluids with magnets (typically lubrication oil, coolants and fuel oils), because there is no filter medium. Commercial devices called "magnetic filters" are sold, but the name reflects their use, not their mode of operation. 


\section{Types of filtration}

Based on the mechanism, three types of the filtration are known [3]. These are:

\subsubsection{Surface filtration}

Surface filtration is a screening action by which pores or holes of the medium prevent the passage of solids. The mechanisms, straining and impingement are responsible for surface filtration. For this purpose, plates with holes or woven sieves are used. Example is cellulose membrane filter.

\subsubsection{Depth filtration}

In depth filtration, the filtration mechanism retains particulate matter not only on the surface but also at the inside of the filter. This is aided by the mechanism entanglement. It is extensively used for clarification. Examples are ceramic filters and sintered filters [4] and cross flow filtration [5].

\subsubsection{Advances in sintered metal filters}

Filtration technology utilizing sintered metal media provides excellent performance for separation of particulate matter. Sintered metal filter media are widely used in the chemical process, petrol-chemical and power generation industry. Advances in filtration technology include the development of continuous processes to replace old batch process technology. Liquids/solids filtration using conventional leaf filters is messy and hazardous to clean and require extended re-circulation time to obtain clean product.

Traditional gas/solids separation system such as cyclones, electrostatic precipitators and disposable filters are being replaced by sintered fibre filtration systems. Sintered metal filter should be operated within the design parameters to prevent premature blinding of the media due to fluctuations in process operations. Use of flow control assures the filter will not be impacted with a high flow excursion. Filter efficiency increases as the filter cake forms. The cake becomes the filter media and the porous media acts as a septum to retain the filter cake. Filter cakes can be effectively washed in-situ and backwashed from the filter housing. The major active component in traditional depth filtration is diatomaceous earth, which has several major problems. First, it is difficult to dispose of because it does not decompose. Second, it can cause symptoms similar to coal miners "black lung" disease when inhaled over long periods of time. In the United States this problem can be overcome by using cross flow filtration. The main benefit of cross flow filtration is that it uses a membrane with an absolute pore size to clarify alcohol without the need for media to act as the sieve for removal of particles from alcohol [6].

\subsubsection{Cross flow filtration}

An example of cross flow filtration is nanofiltration which is a recent membrane filtration process used most often with low total dissolved solids water, with the purpose of softening and removal of disinfection byproduct precursor such as natural organic matter and synthetic organic matter. Nanofiltration ranges somewhere between ultra filtration and reverse osmosis. The nominal pore size of the membrane is typically below 1 nanometer, hence so called nanofiltration. Nanofilters membranes are typically rated by molecular weight cutoff than nominal pore size. The trans-membrane pressure required is considerably lower than the one used for reverse osmosis, reducing the operating cost significantly. However, nanofitration membranes are still subject to scaling and fouling and often modifiers such as anti-scalants are required for use [7].

\section{Ultra filtration}

Ultra- filtration is a pressure driven membrane transport process that has been applied, on both the laboratory and industrial scale. Ultra filtration is a separation technique because labile streams of biopolymers (proteins, nucleic acids and carbohydrates) can be processed economically, even on a large scale, without the use of high temperatures, solvents, etc. Shear denaturation can be minimized by the use of low shear (e.g., positive displacement) pumps [8]. Infusion solvents, serum, vaccines and plasma are only some of the products of the pharmaceutical industry that are produced to meet the highest standard concerning quality and purity.

Ultra filter offers systems which have been developed to match the requirement of the pharmaceutical industry and biotechnology for numerous applications. In order to ensure consistent quality of the final products the entire manufacturing process must be free from any contamination. The aim is reached in a reliable, safe and cost efficient way by ultrafilter membrane filters. The following types of ultra filtration membranes are used prominently. These are asymmetric skinned membranes made from synthetic polymers by the "phase inversion" methods. Inorganic membranes, utilizing inorganic porous supports and inorganic colloids, such as $\mathrm{ZrC}^{*} 2$ or alumina with appropriate binders [9].

\subsubsection{Recent \& developing application of ultra filtration}

Ultra filtration is becoming a powerful separation tool for the rapidly growing biotechnology industry. Examples are cell harvesting, depyrogenation of injectable drugs, and enzyme purification. Ultra filtration also 
offers some important advantages over centrifugation for harvesting of bacteria. These advantages are such that the asymmetric character of ultra filtration membranes renders them less prone to clogging by cells and debris than micro porous filters. Plasma product processing is another promising application of ultra filtration. When human plasma is fractionated by the Cohn process or some new methods, a need arises for concentration of the important protein fractions (albumin \& globulins) or for removal of alcohol and salt from these fractions. This can be conveniently accomplished by ultrafiltration.

\section{Cake filtration}

By this filtration mechanism, the cake accumulated on the surface of the filter is itself used as a filter. A filter consists of a coarse woven cloth through which a concentrated suspension of rigid particles is passed so that they bridge the holes and form a bed. Example is cake made from diatomite. This cake can remove sub micrometer colloidal particles with high efficiency. The fermentation process shown in Figure 1 shows how ultrafilters are used in this application while Table 1 shows application of the various filters with their pore sizes.

\section{Theory of filtration}

Depending on dispersing medium filtration is divided in two parts; Gas filtration and liquid filtration.

\subsubsection{Gas filtration theory}

Gas filtration includes filtration of aerosol and lyosol. Membrane filters and nucleopore filters are based on three mechanisms. The first is the diffusion deposition where the trajectories of individual small particles do not coincide with the streamlines of the fluid because of Brownian motion. With decreasing particle size the intensity of Brownian motion increases and, as a consequence, so does the intensity of diffusion deposition. The second is the direct interception where the mechanism involves the finite size of particles. A particle is intercepted as it approaches the collecting surface to a distance equal to its radius. A special case of this mechanism is the so-called sieve effect, or sieve mechanism. The third is the inertial deposition where the presence of a body in the flowing fluid results in a curvature of the streamlines in the neighbourhood of the body. Because of their inertia, the individual particles do not follow the curved streamlines but are projected against the body and may deposit there. It is obvious that the intensity of this mechanism increases with increasing particle size and velocity of flow. In electrostatic deposition both the particles and the fibers in the filter may carry electric charges. Deposition of particles on the fibers may take place because of the forces acting between charges or induced forces [10].

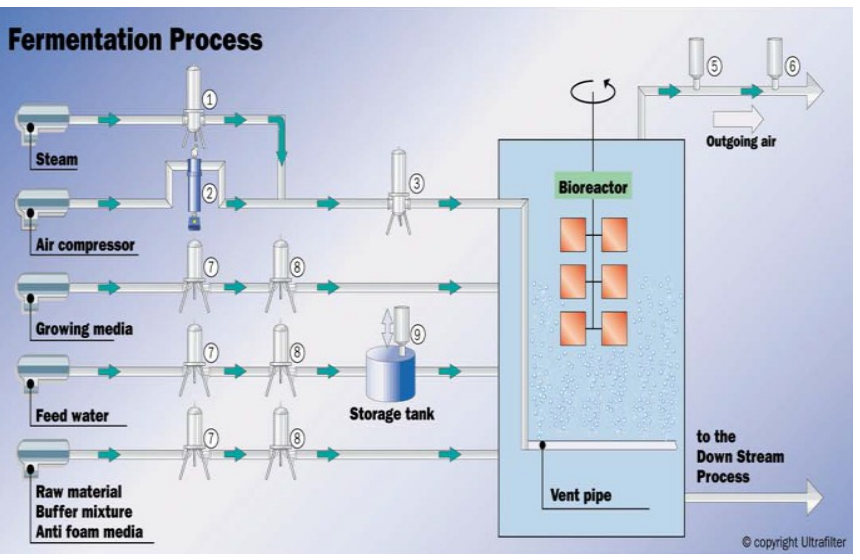

Fig 1: Fermentation process in the pharmaceutical industry

\subsubsection{Liquid filtration theory}

The term solid-liquid filtration covers all processes in which a liquid containing suspended solid is freed of some or the entire solid when the suspension is drawn through a porous medium [11]. This is better explained by:

Kozeny-Carman equation:

1.dv/A.dt $=\Delta \mathrm{P} / \mathrm{r} \mu(1+\mathrm{L})$ 
Table 1: Showing applications of the various ultrafilters with their pore sizes

\begin{tabular}{llll}
\hline $\begin{array}{l}\text { Filter } \\
\text { no. }\end{array}$ & Application & Recommended ultrafilter filter & Pore size \\
\hline 1 & & & $1-25 \mu \mathrm{m}$ \\
2 & Steam filter for sanitation & Ultra P-GS & $0.01 \mathrm{mg} / \mathrm{m}^{3}$ \\
3 & Pre filter (air) & Ultradepth SMF & $0.01 \mu \mathrm{m}$ to 99.99998 \\
& Sterile filter & Ultradepth II P-SRF & $0.1-0.2 \mu \mathrm{m}$ \\
& & Ultrapolymem P-PF-PP & $0.1-0.2 \mu \mathrm{m}$ \\
$5+6$ & De-aeration of fermentation exhaust air & Ultrateflomem P-PF-PT & $0.01 \mu \mathrm{m}$ up to $99.999 \%$ \\
9 & venting of storage tank & Ultradepth II P-BE & $0.1-0.2 \mu \mathrm{m}$ \\
& & Ultrapolymem P-PF-PP & $0.1-0.2$ \\
7 & Prefiltration & Ultrapolyplea P-PP & $0.2-1 \mu \mathrm{m}$ \\
& & Ultrapolyplea P-PP100 & $0.8-2.4 \mu \mathrm{m}$ \\
\hline
\end{tabular}

where, $\mathrm{A}=$ filter area; $\mathrm{V}=$ total volume of filtrate delivered; $\mathrm{t}=$ filtration time; $\Delta \mathrm{P}=$ pressure drop across cake and medium; $r=$ specific cake resistance; $\mu=$ filtrate viscosity; $1=$ cake thickness; $\mathrm{L}=$ thickness of cake equivalent to medium resistance.

\subsubsection{Limitations of Kozeny-Carman equation}

This equation does not take into account of the fact that depth of the granular bed is lesser than the actual path traversed by the fluid. The actual path is not straight throughout the bed, but it is sinuous or tortuous [12].

\subsubsection{Poiseulle's law} capillaries.

This Law considered that filtration is similar to the streamline flow of a liquid under pressure through

$$
1 . \mathrm{A} / \mathrm{dv} \cdot \mathrm{dt}=\Delta \mathrm{P} / \mu\left(\mathrm{R}_{\mathrm{M}}+\mathrm{R}_{\mathrm{C}}\right)
$$

Cake resistance $\mathrm{RM}=\sigma \mathrm{W} / \mathrm{A}$

Specific cake resistance: $\sigma=\sigma^{\prime} \Delta \mathrm{P}^{\mathrm{s}}$

The filter resistance is much less than the cake resistance. $\mathrm{Rc}<<\mathrm{Rm}$

Therefore, rearranging equation (1):

$$
\mathrm{A} / \mathrm{dv} \cdot \mathrm{dt}=\Delta \mathrm{P} / \mu\left(\sigma^{\prime} \Delta \mathrm{PSWA}\right)
$$

where $\mathrm{V}=$ filtrate volume, $\mathrm{A}=$ Filter area, $\mathrm{t}=$ Time, $\Delta \mathrm{P}=$ Pressure driving force, $\mu=$ Broth viscosity, $\mathrm{W}=$ Mass of filter, $\mathrm{R}=$ resistance, $\sigma=$ specific cake resistance, $\mathrm{S}=$ compressibility factor.

\section{Water purification for pharmaceutical industry}

There are two relevant standards concerning the water quality for the pharmaceutical and biotechnology industry:

1-Water for injection (WFI)-This water must meet the highest level of requirement. It is used for the adjustment of product concentration, for the production of WFI steam, and for the cleaning of ampoules or vials.

2-Purified water (PW) or deionized water (DI-water)-This is mainly used for cleaning of bottles and ampoules or as a supply for fermenters. It is also an addition to lotions, creams and used in the production of purest steam for sterilization. For the production of these water qualities Ultrafilter offers the asymmetrical polyethersulfone membrane with retention rates down to 40 Nanometers $(=0.04 \mu \mathrm{m})$. This filter medium is characterized by substantial high flow rates and a low filtration costs. Figure 2 shows a typical process of sterile water treatment while Table 2 shows the ultrafilters used for water treatment.

\section{Filtration of chemicals and solvent in the pharmaceutical industry}

Apart from water, chemicals and solvents are important complementary materials in the production of pharmaceutical and biotechnical products. Alcohols, acetones and methylene chloride are the most frequently used products. All these chemicals are aggressive and therefore the filtration system has to be adjusted to meet these requirements as well as the usual filtration 


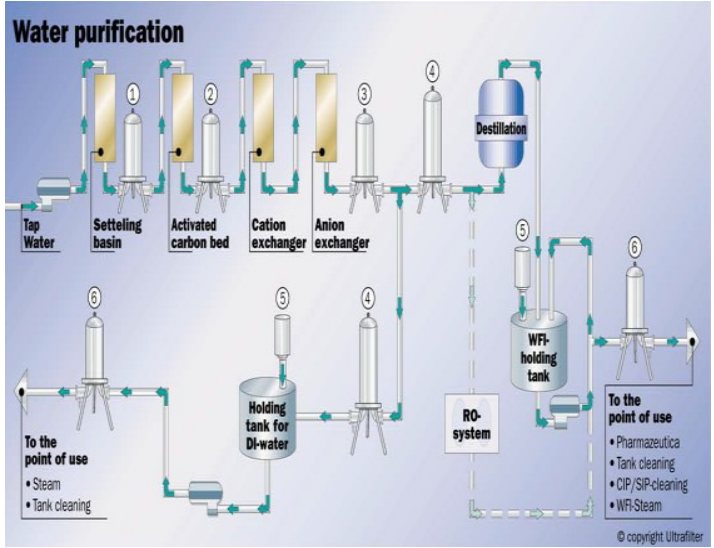

Fig 2: Refining of water in the pharmaceutical industry

Quality. Figure 3 shows the filtration of chemicals from raw materials to the final product which the consumer will receive. Table 4 shows Ultrafilter products which are recommended for filtration to ensure a highly efficient and consistent quality, even in the event that the quality of the raw materials should fluctuate.

Table 2: Showing utrafilters used for water treatment

VIII. Sterile filling of pharmaceutical and biological products

Filtration by membrane guarantees sterility in filling pharmaceutical products. This ensures an efficient

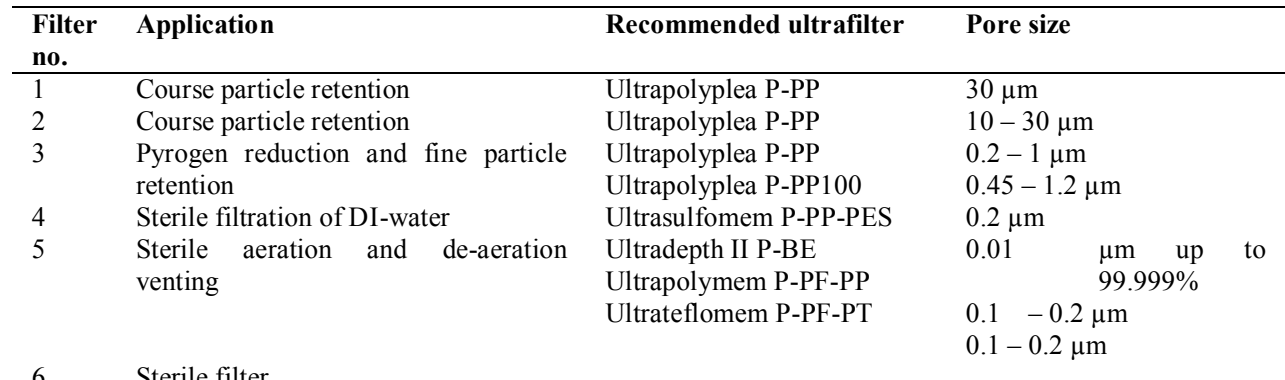

retention of pyrogenes, living microorganisms and particles at a maximum flow rate. As a prefilter for protecting the membrane ultrapolyplea ${ }^{\circledR}$ depth filters are used. These filter elements have a high dirt hold capacity and low extractables. In storage and holding tanks, hydrophobic ultrafilter aeration and de-aeration filters guarantee sterile conditions for storing the sensitive products and exclude a contamination by the ambient air. Figure 4 shows the process of sterile filling and the ultrafilter products designed for it. Table 4 shows ultra filter products recommended for filtration of solvent and chemicals.

\section{Filter media}

The filter medium acts as a mechanical support for the filter cake and it is responsible for the collection of solids [13].

\subsubsection{Materials used as filter media}

Different types of materials used as filter media are presented in Table 2. Woven materials such as felts or cloths: woven material is made of wool, cotton, silk \& synthetic fibers etc. are used. Synthetic fibers have greater chemical resistance than wool or cotton. The choice of fiber also depends on the physical state \& chemical constitution of the slurry.

\section{Choice of filter media}

The choice includes mainly of two types:

\subsubsection{Monofilament woven cloth.}

The yarns of a monofilament fabric are not only impermeable but also fairly smooth and cylindrical. Orifice analogy and drag theory approaches have been the most successful in predicting the resistance of these materials to fluid flow [14]. 
1.10.2 Multifilament woven cloth.

The chief difficulty encountered when dealing with multifilament media is the highly

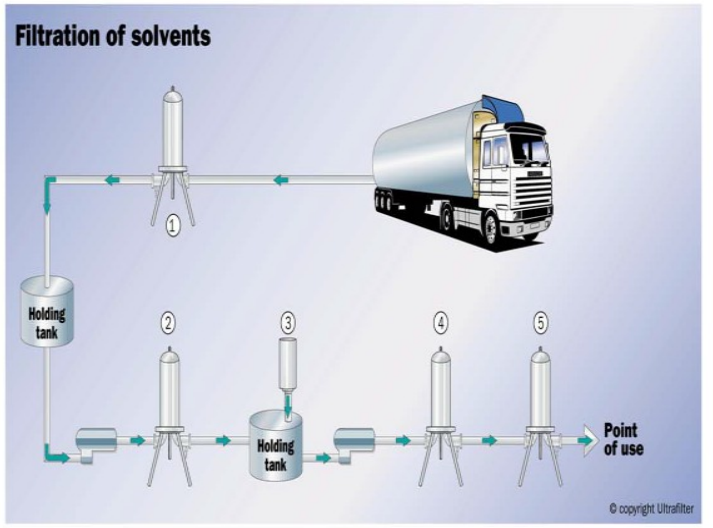

Fig 3: Schematic diagram Filtration of solvents/chemicals

Complex geometry of the fibers and yarns that make up the cloth. Even in a fabric of apparently simple weave and construction, such as a plain-weave, continuous filament cloth, some of the flow takes place in the highly tortuous channels present in the yarns [15].

\subsubsection{Perforated sheet metal}

Stainless steel plates have pores which act as channels as in case of Meta filter. Bed of granular solid built up on a supporting medium: examples of granular solids are gravel, sand, asbestos,

Table 4: Ultra filter products recommended for filtration for solvent and chemicals

\begin{tabular}{llll}
\hline Filter no. & Application & Recommended ultrafilter & Pore size \\
\hline 1 & Prefilteration of the raw materials & Ultrapolyplea P-PP & $30 \mu \mathrm{m}$ \\
2 & Fine filteration & UltrapolypleaP-PP & $5-10 \mu \mathrm{m}$ \\
& & Ultrapolyplea P-PP 100 & $3-10 \mu \mathrm{m}$ \\
3 & Container de-aeration & Ultradepth II P-PE & $0.01 \mu \mathrm{m}$ up to $99.999 \%$ \\
& & Ultrateflomem P-PF-PT & $5 \mathrm{~m}$ \\
& & Ultraployplea P-PP 100 & $5-10 \mu \mathrm{m}$ \\
4 & Prefiltration & Ultrapolyplea P-PP 100 & $0.8-2.4 \mu \mathrm{m}$ \\
5 & Sterile filtration & Ultrasulfomem P-PF-PES & $0.1-0.2 \mu \mathrm{m}$ \\
& & Ultrapolymem P-PF-PP & $0.1-0.2 \mu \mathrm{m}$ \\
& & Ultrateflomem P-PF-PT & $0.1-0.2 \mu \mathrm{m}$ \\
\hline
\end{tabular}

paper, pulp \& kieselguhr. Prefabricated porous solid unit: sintered glass, sintered metal, earthenware and porous plastics are material used for fabrication. Membrane filter media: it includes surface \& depth type of cartridges.

\section{Criteria for choice of filter medium}

There are three criteria for choice of filter medium:

1. Size of particle retained by the medium.

2. The permeability of the clean medium

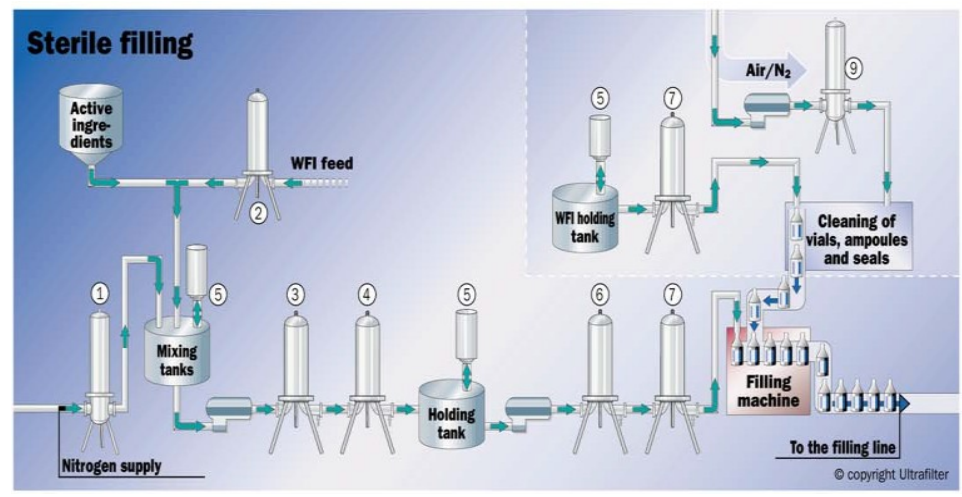

Fig 4: Schematic diagram of sterile filling assembly in the pharmaceutical industry 
3. The solid holding capacity of the medium and the resistance to fluid flow of the used medium [16]. Some examples of filter media used in the pharmaceutical industry are stainless steel (plain, twill and Dutch type); anthracite filter media as in water purification [17].

\section{Measurement of pore size \& particle retention}

In some cases, the desirable component in the slurry is the liquid, which may be required in clarified form e.g., beverage filtration; here the choice of deep-bed elements of precoated candles of large solids-holding capacity may be indicated. While, where the solids are valuable, a

Table 5: Recommended ultrafilters for sterile pharmaceutical production

\begin{tabular}{|c|c|c|c|}
\hline Filter no. & Application & Recommended & Pore size \\
\hline \multirow[t]{2}{*}{1} & Sterile filteration of nitrogen $\left(\mathrm{N}_{2}\right)$ & Ultradepth II P-SRF & $\begin{array}{lrlr}0.01 \quad \mu m & \text { up } & \text { to } \\
99.99998 \%\end{array}$ \\
\hline & & Ultrapolymem P-PF-PP & $0.1-0.2 \mu \mathrm{m}$ \\
\hline 2 & Sterile filtration & UltrasulfomemP-PF-PES & $0.1-0.2 \mu \mathrm{m}$ \\
\hline $3+6$ & Prefilter & Ultrapolyplea P-PP 100 & $0.8-2.4 \mu \mathrm{m}$ \\
\hline $4+7$ & Sterile filter & Ultrasulfomem P-PF-PES & $0.1-0.2 \mu \mathrm{m}$ \\
\hline 5 & & Ultrateflomem P-PF-PT & $0.1-0.45 \mu \mathrm{m}$ \\
\hline 8 & $\begin{array}{l}\text { Sterile filtration of water for the cleaning of } \\
\text { vials/ampoules }\end{array}$ & Ultrasulfomem P-PF-PES & $0.1-0.2 \mu \mathrm{m}$ \\
\hline \multirow[t]{2}{*}{9} & Nitrogen/air for drying of vials/ampouls & Ultradepth II P-SRF & $\begin{array}{l}0.01 \mu \mathrm{m} \\
99.99998 \%\end{array}$ \\
\hline & & Ultrapolymem P-PF-PP & $0.1-0.2 \mu \mathrm{m}$ \\
\hline
\end{tabular}

sieve like mechanism is favored, so that information about the pore size of the medium may be of more direct use in media selection. The pore structure of the medium will determine the feasibility of a separation. The pore size of a medium particularly for filters of the edge, simple wire or monofilament type is of use in deciding the upper limit of aperture size required by a particular process. In filters composed of random fibers, sintered or porous elements, staple or natural fiber cloths, the mean pore size will have less significance and use in predicting media behaviour. In certain cases, the geometry of septum allows direct measurement of aperture or pore size. In random situation, where complex weave pattern produce a distribution of pore sizes, such as a bubble point test or a permeability test are used [18].

\section{Filter aids}

Filter aids form a surface deposit which screens out the solids and also prevents the plugging of the supporting filter medium. They are also used as filter media in recoat filtration. . The ideal characteristics of filter aid materials are chemically inert to the liquid being filtered \& free from impurities, low specific gravity (so that filter aids remain suspended in liquid), porous rather than dense (so that previous cake can be formed) and recoverable [17].

\section{Filtration equipments}

Different forms of equipment are employed for filtration. The factors which should be considered, while selecting the equipment and operating conditions on the basis of Shirato [19] (1978) are: (1) Material related-that is properties of the fluid which include viscosity; nature of the solids which are particle size, shape, size distribution etc; concentration of solids in suspension; quantity of material to be handled (2) Equipment \& process related, that is, flow rate; the limit to size of particles passing through the filter should be known Equipment should be sterilized by heat, radiation or gas and economical.

\subsubsection{Belt filter:}

The belt filter is an industrial machine, used for solid/liquid separation processes, particularly the dewatering of sludge's in the chemical industry, mining and water treatment. The process of filtration is primarily obtained by passing a pair of filtering cloths and belts through a system of rollers.

\subsubsection{Operation of belt filter}

The feed sludge to be dewatered is introduced from a hopper between two filter cloths (supported by perforated belts) which pass through a convoluted arrangement of rollers. As the belts are fed through the rollers, water is squeezed out of the sludge. When the belts pass through the final pair of rollers in the process, the filter cloths are separated and the filter cake is scraped off into a suitable container. 


\subsubsection{Improvements in belt filters}

1. The effectiveness of the operation can be increased by creating a pressure difference across the filter cloth. The filter cloth is directed though a zone where either pressure or vacuum pushes water from the filter cloths and ultimately to drain.

2. The sludge can be combined with a filter aid or flocculants which help the filtration process and reduce blinding of the filter cloth.

3. Filter cloths can be cleaned throughout the operation of the process by means of water sprays positioned on the return section of the belt.

\section{Bag filter}

Filter Bags are made of felt material, which has the advantage of providing three dimensional filter media and offers both, a surface and depth filtration effect. They are available in different types of filter media in different ratings. Polypropylene filter bag offers a broad range of chemical compatibility and is suitable for many applications. Polyester filter bags are suitable for high temperature with compatibility for acids and petroleum based fluids. Bag filter is mainly used for the preparation of adhesives, fruit juices, petroleum products and high viscosity fluids [20].

\subsubsection{Advances in bag filtration system}

Innovations in both the filter housing and the bag filtration media now make it possible for Eaton bag filters to be used in applications that previously required more costly types of filtration equipment. Eaton filtration's new patent pending HAYFLOW(TM) filter element combines the best of both bag and cartridge filters into one single filtration element for enhanced performance [20].

\section{Self cleaning filters}

Various methods for cleaning the filters are manual, mechanical and self-cleaning filter systems. Manual cleaning is done by jet of water. Sometimes the filters have to be soaked in chlorine or detergent. Mechanical cleaning is done by brush or knife. Automation minimizes disposable waste and labour costs. Self cleaning filter system reduces product loss and requires minimal operator intervention. Examples are magnetically coupled industrial filters $\mathrm{MCF}$, self -cleaning filters and tubular backwashing filters including clearamine and reactogard filters [20]. There are three main types of flushing. 1- Direct flushing method causes the water to flow at high velocity at a tangent to the screen and removes the filter cake from filter element. 2 Backflushing is used for flushing sand and gravel filters. In these filters the direction of filtration is from top to bottom. During flushing, water flows in the opposite direction. The water pressure which operates from bottom to top causes suspension of the bed inside filter. Light particles are washed out with water. The particles which are heavy stay in the vessel. At the end of flushing the sand settles \& recreates the filter bed. Forced back flushing filters is a suction device which scans the screen and causes a local forced back flushing.

\section{Conclusion}

Filtration is an important operation in the pharmaceutical industry. Hardly there is any part of a pharmaceutical industry that is not touched. From production unit to "in process" to chemical and research laboratories to the purification of water for sterile and non sterile products, all of which involve some form of filtration in order to achieve a good manufacturing practice (GMP). Thus, a self cleaning filter reduces product waste and minimizes time for the attainment of the GMP. Sintered metal filters which is discussed here is a continuous type of process which replaces old batch process. Many patent filters are commercially available which combine the bag and cartridges types. In this way, by using innovative filter equipments we can reduce labour cost, get better quality products and maximize the yield in lesser time.

\section{Acknowledgements}

Authors wish to thank and acknowledge the immense contributions of the Donaldson Ultrafilters ${ }^{\circledR}$ Company and Parksan Filter Ltd. producers and marketers of various types of filters and water equipments for making their literatures available online for us.

\section{References}

[1] R. Patel, S. Devarshi, G. Bhupendra, P. Patel and M. Patel, Overview of industrial filtration technology and its applications. Indian journal of Science and Technology. Vol. 3 No. 10, 2010, 1121-1127.

[2] K. Sambhamurthy, Filtration: In pharmaceutical engineering, New age international Ltd. 12, 2005,248

[3] J. Matteson, Filtration principles and practices; gas filtration theory in 2nd edition (ed.) 1987, pp28-32.

[4] E. A. Stephan, Use of genetic algorithms as an aid in modeling deep bed filtration. Comp. Chem. Engg. 27, $2003,281-292$.

[5] Mott corp. Adv. Filtration Separation Technol. 1999, 512-520.

[6] J. Hillie, Nanotechnology and the challenge of clean water. Nature Nanotechnol. 2, 2007, 663-664. 
[7] R. L. Goldsmith, D. A. Roberts and D. L. Burre, Ultrafiltration of soluble oil wastes. Water Pollution Control. 46(9), 1974, 2183

[8] C. A. Smolders, Ultra filtration membranes \& applications, plenum, 1980, New York.

[9] L. G. Wilson and P. Cavanagh, Principle of inertial impaction, Atom, Environ. 3, 1969, 597-605.

[10] C. R. Melia and W. J. Weber, Liquid filtration theory: In physic-chemical processes for water quality control.. Wiley interscience, 1972, New York, 61

[11] P. Chowdiah, D. Wasan and D. Gidaspow, Electro kinetic phenomena in the filtration of colloidal particles suspended in nonaqueous media. All India chemical eng. J, 1981, 27, 975.

[12] C.V. S. Subramanyam, T. J. Setty, S. Suresh and K. V. Devi, Filtration In pharmaceutical engineering principles and practices, Vallabh prakashan, 2005, Delhi, 9, 248-250.

[13] A. Rushton, Solid-liquid filtration \& separation technology. Filter Media. 2008, 209-236.

[14] L. C. Wardsworth, Handbooks of nonwoven filter media. J. Engineered Fibers Fabrics. 2(1), 2007, 61-63.

[15] D. B. Purchas, Filters media: Industrial filtration of liquids, 2001, Leonard hill, London.

[16] J. A. Hunt, Filtration media: Making the right choice. Water Conditioning Purification Magazine, 2001

[17] V. H. Lach and A. E.Wright, Recent advances in filtration. Chemical Health Safety. 11(2), $2004,33$.

[18] M. Shirato, Filtration in chemical process industry in chemical engineer's handbook. $4^{\text {th }}$ (Ed); Tokyo, 1978, p1 131

[19] M. Shirato, filtration in chemical process industry in chemical engineer's handbook. $4^{\text {th }}($ Ed), 1978, Tokyo, 1131

[20] Parksanfilter, Literature from Parksan Filters Company, 2010. 